\title{
Disciplinary emotions in imperialistic interdisciplinarity
}

\author{
Salmela, Mikko Erkki Matias
}

Routledge

2018

Salmela , M E M \& Mäki , I U 2018 , Disciplinary emotions in imperialistic interdisciplinarity . in U Mäki , A Walsh \& M Fernández Pinto (eds), Scientific Imperialism : Exploring the Boundaries of Interdisciplinarity . Routledge Studies in Science, Technology and Society, Routledge, Abingdon, pp. 31-50.

http://hdl.handle.net/10138/233789

acceptedVersion

Downloaded from Helda, University of Helsinki institutional repository.

This is an electronic reprint of the original article.

This reprint may differ from the original in pagination and typographic detail.

Please cite the original version. 


\title{
Disciplinary emotions in imperialistic interdisciplinarity
}

Mikko Salmela \& Uskali Mäki

Academy of Finland Centre of Excellence in the Philosophy of Social Sciences

University of Helsinki

Post-print / accepted draft

In Mäki, Uskali; Fernández Pinto, Manuela \& Walsh, Adrian (Eds.) (2018). Scientific Imperialism: Exploring the Boundaries of Interdisciplinarity. Routledge.

ISSN: 9781138059344 (Hardback) / 9781315163673 (eBook)

\begin{abstract}
Emotions are an important yet largely neglected aspect of scientific work. Little is known about their role in the constitution and maintenance of disciplines and disciplinary identities. We present a theoretical account of disciplinary emotions and highlight their role in interdisciplinary interaction, focusing on scientific imperialism. We argue that disciplines are institutions with epistemic and organizational aspects that come together in internalized and enacted disciplinary cultures and identities that provide the intentional and psychological background for the emergence of disciplinary emotions. These are felt in the social identity of a scholar for reasons that relate to the epistemic and organizational aspects of the discipline. In interdisciplinary interaction, disciplinary emotions - such as feelings of superiority and inferiority, confidence and pride, envy and jealousy, or anger and fear - motivate proponents and opponents of scientific imperialism alike. We propose that imperialistic disciplines such as economics and evolutionary biology motivate their actions by second-order judgments about the interdisciplinary applicability of their first-order theoretical and methodological principles. Finally, we suggest that the justification of disciplinary emotions in the context of scientific imperialism should be evaluated in terms of their adaptiveness in promoting epistemically desirable ventures in scientific research.
\end{abstract}




\section{Introduction}

Emotions are an important aspect of scientific work, yet they are still largely neglected in the research on science. Their role in shaping scientific observation and discovery as well as the justification, revision and rejection of old convictions and new conjectures has nevertheless received some attention in sociology, philosophical epistemology, and philosophy of science, beginning from the early writings of Ludwik Fleck $(1935,1936)^{1}$. He argued that collective emotions and moods are important in scientific activity conducted by 'thought collectives' with distinct 'thought styles' - shared cognitive frameworks characterized by common research problems, evaluative standards, methods, techniques, and literary styles. Fleck, who believed that "there is no emotionless state or pure rationality as such" $(1935,49)$, suggested that 'thought styles' evoke and maintain a collective mood that "produces the readiness for an identically directed perception, evaluation, and use of what is perceived" $(1936,101)$. Recent research supports the view that emotions indeed have these roles in scientific perception and evaluation. Thus, Jack Barbalet (2011) suggests that scientific aesthetics, learned during training in a specific research community, guides scientific perception and gives rise to aesthetic pleasure when a particular research episode corresponds with the emotionally-laden values of the research community. Several philosophers have also highlighted the functions of emotions as detectors of salience and in epistemic justification (Elgin, 2008; Hookway, 2008; Tanesini, 2008; de Sousa, 1987). Scientists' own descriptions of research process confirm these roles of emotions in science (e.g. Koppman, Cain, \& Leahey, 2015).

Yet these reflections on emotions in social cognition overlook another important way in which emotions are involved in scientific practice. This is their role in the production and reproduction of scientific disciplines and the associated disciplinary identities of scientists. Indeed, if we understand disciplines along the lines of Fleck's 'thought collectives' with 
distinct 'thought styles' as their epistemic cores, the affectivity of thought styles brings emotion into the heart of disciplines.

What is important for our purposes is that emotions not only shape the internal social lives of disciplines, but also make a significant difference for their external relations. This is where interdisciplinarity enters the stage. The importance of disciplinary emotions has increased together with the growing stress on interdisciplinarity in research. In interdisciplinary collaboration, scholars from different disciplines are supposed to negotiate the concepts, methods, and theories applied in their joint projects. These situations trigger various emotions such as those that arise if some participants of an interdisciplinary project feel that they are treated unjustly by participants from another discipline or disciplines. Another set of contexts in which disciplinary emotions emerge consists of non-collaborative asymmetric relations characterized by intellectual or institutional expansion across disciplinary boundaries. Versions of such scientific imperialism include the phenomenon in which one discipline seeks to 'invade' a domain that is already 'occupied' by another discipline with it is own concepts, methods, and theories. In these encounters among disciplines, disciplinary emotions motivate both the proponents and opponents of scientific imperialism.

We begin by analyzing the nature of disciplines, which is crucial for understanding the social identification of individual scholars. This background allows us to analyze disciplinary emotions as group-based emotions of a specific kind that are both causally and rationally tied to disciplinary identities. We then proceed to examine the role of different disciplinary emotions in interdisciplinary interaction, and in scientific imperialism in particular, elucidating the situations in which these emotions emerge and the kinds of behavior they motivate. We suggest that the internal justification of imperialistic disciplinary emotions as motivational forces derives from the epistemic core of the discipline that contains 
second-order judgments about the superiority or some other special expediency of the imperialistic discipline in relation to others. However, the rational justification of such disciplinary emotions - as well as those that are prompted in the target disciplines of scientific imperialism -- depends on their adaptiveness in promoting epistemic progress in scientific inquiry at large.

\section{What are disciplines?}

There is a wide agreement on the nature of disciplines as normative institutions whose two main aspects, the epistemic and the organizational, always go together (Mäki, 2013). Yet there is a third aspect: disciplinary cultures and identities that reflect the ways in which members of a discipline internalize and enact its epistemic posits and social arrangements.

Considering its epistemic aspect, a discipline is a more or less convergent system of concepts, methods, theories, questions, established facts, canonical convictions, standards of evaluation, and styles of argumentation. The 'more-or-less' here is to be taken literally. Some disciplines are epistemically and methodologically more plural and diverse than others, involving different schools and traditions and fashions that coexist (in peace or in conflict), as in the case of sociology and human geography; whereas other disciplines - especially if they are guided by an ideal of unity - seek to suppress diversity, with rival schools perhaps actively trying to undermine the credibility of each other, as was the case for instance between analytic and continental philosophy from early $20^{\text {th }}$ century until recently.

Epistemic rivalries within disciplines often relate to struggles for organizational positions, resources and power. The organizational aspect of disciplines consists of ingredients such as university departments, endowed chairs and other academic and 
administrative positions, educational programs and degrees, certifications and affiliations, research centers and institutes, scholarly associations, conferences, and journals. Until recently, the structure of academic departments in many cases used to correspond - roughly with the structure of disciplines on a one-to-one basis. However, things are changing along with cuts of public funding and increased demands of cost efficiency in higher education. These trends have inspired another trend, that of turning departments into large multidisciplinary units, especially in many European universities. While this development in administrative structure is often justified in terms of promoting interdisciplinarity (on top of saving in administration costs), it may also serve to shake the disciplinary foundations of interdisciplinarity by putting pressure on the organizational autonomy of disciplines (e.g Radder 2010).

If the two aspects of a discipline, the epistemic and the organizational, always coexist and entangle with each other, it might be futile to ask which one is primary. In part, this depends on the perspective we take on disciplines. From an internal perspective, the epistemic aspects of a discipline constitute its core even if this core is liable to change. Advances in theories, methods, and concepts drive the development of a discipline and motivate the inception of new organizational forms and forums such as conferences, associations, networks, and journals rather than the other way round. A discipline obviously needs some organizational 'bodies' in which its epistemic 'mind' can operate its two main functions of research and education. However, the organizational structure can be flexible as long as it allows for sufficient autonomy to the discipline. Evidence from studies on socialization to disciplinary identities supports the primacy of the epistemic aspect of disciplines as well. Disciplinary cultures are maintained and perpetuated by means of identification with disciplinary norms and ideologies that transcend the organizational settings of academic departments (Parry, 2007; Henkel, 2000). 
Exploiting the resources of Raimo Tuomela's (2007) theory of social ontology, we may characterize disciplines as social institutions with an ethos: a set of constitutive goals, values, beliefs, standards, norms, practices, and/or traditions that give scholars both motivating and justifying reasons for their disciplinary activities. The constitutive ethos of a discipline consists of a distinct set of epistemic and methodological principles, standards, norms, and values that the members of the discipline seek to satisfy and promote in their activities as group members affiliated with a discipline. Disciplines host several organizations such as university departments, research institutes, or academic societies, and their particular members occupy task-roles such as a professor or a president in those organizations. Yet it is sometimes possible to conduct disciplinary research (in some disciplines) even without occupying a task-role in the academia by following the relevant disciplinary norms in one's activities. Indeed, most leading European philosophers until the $19^{\text {th }}$ century did not work at universities. This is one reason for not identifying disciplines with their organizational aspects even if some organizational structure is necessary for the maintenance and dissemination of the disciplinary ethos and for validating sufficient mastery among the novices.

Another main requirement of social institutions in Tuomela's theory is their collective acceptance by group members. All disciplines have some foundational norms and ideas on which those affiliated with the discipline more or less agree. Again, the 'more-orless' is important here. The degree of agreement varies from discipline to discipline (biology to economics, for example) and from some parts of a discipline to its other parts (say theoretical to methodological aspects). Intra-disciplinary diversity is consistent with collective acceptance and agreement when it is based on acknowledged complementarity, with research fields focusing on their sub-domains, addressing their specific problems and applying their distinctive techniques in harmony with one another. Or diversity can be based on rivalry and disagreement, when alternative perspectives are offered in competition or even in conflict 
with one another. For example, those agreed-upon norms can be methodological and concern legitimate ways of making and justifying arguments, collecting evidence, forming and testing hypotheses, and so on, even if there are disagreements on substantial theoretical issues. If there is no agreement on deep theoretical convictions or on the appropriate methods of adjudicating disputes between different positions, we can talk about different schools with partly dissimilar ethoses within a discipline. Yet different schools typically share roughly the same domain of phenomena, research problems, and recognized classic texts that define those problems, and thereby the ambit of the discipline. The shared ethos of a divided discipline is founded on those shared problems and classic texts rather than on the present standards and theories. Yet, at least typically, there is a more substantial agreement on epistemic and methodological criteria within a discipline.

In addition to epistemic and organizational aspects, disciplines have a third aspect which is often attributed to people working in the discipline. This aspect focuses on the ways in which scholars internalize and enact the epistemic posits and social arrangements of their discipline. Disciplinary culture comprises epistemic and methodological commitments of the discipline, but also material practices of disciplinary work and social relations of knowledge production in and through which this work is conducted (Schoenberger 2001). All these aspects of disciplinary culture are reflected in a sense of a disciplinary identity in terms of which we understand who we are and what we do in the academic world. Together, disciplinary culture and identity provide an intentional and psychological background for the emergence of what we propose to call disciplinary emotions; pride and fear, envy and jealousy, confidence and insecurity, feelings of disciplinary superiority and inferiority, and other similar emotions that are experienced as members of a discipline on specifically disciplinary grounds. Emotions contribute to the process of disciplinary socialization in interaction with senior colleagues and peers in seminars, workshops and conferences in ways 
that Collins (1998) has analyzed in detail. Consequently, emotions become rational responses to events that are significant from the perspective of the disciplinary identity. The rationality of emotions must here be understood in a Davidsonian sense that refers to their forming a coherent and holistic pattern with an agent's attitudes and other responses, rather than their being mediated by reason or reasoning. ${ }^{2}$

\section{What are disciplinary emotions?}

We next analyze disciplinary emotions as emotions that are felt in the social identity of a disciplinary scholar. Disciplinary emotions are emotions felt for reasons that rationally again, in a Davidsonian sense - relate to the epistemic or organizational aspects of the discipline. The reasons for disciplinary emotions are grounded in the disciplinary ethos, for instance when we evaluate our academic work in research or teaching, or that of our disciplinary colleagues. We feel happy and proud of our (either personal or joint) academic successes; sad and disappointed at our (likewise, personal or joint) academic failures; ashamed if we fail to live up to the core values of our discipline; angry at colleagues who violate those values; and so on. In addition to providing motivating reasons for these emotions, the disciplinary ethos also gives justifying reasons for those emotions within the disciplinary community. However, disciplinary emotions can be felt for organizational reasons as well. Thus, we are shocked if we learn that our university plans to discontinue teaching and research in our discipline, or to reduce its resources substantially, or merge our discipline into a larger academic unit in which its autonomy is seriously jeopardized.

Disciplinary emotions can be either private or shared with others, depending on the context. ${ }^{3}$ These emotions are typically adopted and reinforced in social contexts - lectures, 
seminars, conferences, and debates, both formal and informal - where they are shared with disciplinary colleagues. Collective enthusiasm, admiration, awe, or some other positive shared emotion is experienced when a scholar in a public presentation succeeds in presenting perceptibly original ideas building on previously established research and theorizing. If the presenter fails in this task, in spite of having raised expectations in the audience, the resulting shared emotion is negative; disappointment, dejection, indignation, or annoyance. Examples of private disciplinary emotions include those that we experience as peer reviewers of article manuscripts and research proposals, for instance; or in general reading scholarly works in our field. We are impressed if the manuscript, research proposal, or monograph meets the disciplinary criteria of excellence particularly well, and irritated or disappointed if it fails in light of the same criteria (and especially irritated when it pretends to excel). ${ }^{4}$

Another important distinction in disciplinary emotions concerns their intentional objects. Some disciplinary emotions are felt about the actions of disciplinary colleagues that are evaluated on disciplinary grounds. For instance, admiration, awe, and envy of a new theoretical insight or empirical finding in one's field presuppose understanding and appreciation of the relevance and epistemic value of the discovery, and this understanding emerges from the disciplinary ethos. An additional reason for envy in contrast to admiration and awe is the fact that someone else presented the new insight or finding, which may appear to imply one's inferiority to the other researcher. The involvement of this personal reason does not prevent envy from being a disciplinary emotion because the goal of being given credit for success in one's field is part of one's disciplinary identity even if this goal is shared across all disciplines. Other disciplinary emotions are felt about other disciplines and/or their representatives. These emotions focus on the theories and world views, research styles and techniques, financial resources and academic prestige of those other disciplines. For instance, it has been argued that economists envied and emulated the mathematical methods of 
physicists that they perceived to be superior to the methods of their own discipline, whereas other social scientists have later envied economics for similar reasons (Mirowski, 1992). Emotions of this kind are important in the context of scientific imperialism.

Finally, not all emotions felt in academia are disciplinary. Disciplinary identities are subcategories of academic identities whose constitutive values, norms, standards, and conventions transcend disciplinary boundaries. Accordingly, disciplinary emotions are a subcategory of academic emotions felt in the social identity of an academic. For instance, some criteria in terms of which the activities of professors are evaluated are the same across disciplines and pertain to their role and academic identity as professors. Consequently, emotions about how individuals meet the demands of the non-disciplinary aspects of their academic identity are not disciplinary even if those emotions are pervasive in academia. For instance, all faculty members may feel angry and irritated about their increased administrative tasks after a poorly planned administrative reform. Regardless of how intense these emotions are, they are not disciplinary insofar as their motivating reasons are not disciplinary. Another important group of non-disciplinary academic emotions are those that relate to power relationships and hierarchies within disciplinary communities, such as processes of inclusion and exclusion from an academic conversation or career (Bloch, 2012). Important as these emotions are, they are more or less similar in all disciplines, and therefore generally academic rather than disciplinary. A major shortcoming of previous research on emotions in the academia has been its inability to distinguish disciplinary emotions felt in the social identity of a disciplinary scholar from other academic emotions. 


\section{Disciplinary emotions in imperialist interdisciplinary relations}

Interdisciplinary interaction is an important context for disciplinary emotions. We now move on to examining the role of disciplinary emotions in interdisciplinary relations, and in scientific imperialism in particular. Imperialistic contexts are characterized by dimensions such as disciplinary superiority and inferiority, centrality and marginality, upgrading and downgrading, dominance and autonomy, hegemony and subjugation, admiration and scorn, neglect and engagement, intrusion and resistance, expansion and protection, unification and separation, and various asymmetries in cognitive and other resources. We distinguish between disciplinary emotions typically felt by scientific imperialists, such as feelings of pride, superiority, and confidence; and disciplinary emotions typically felt by those in the imperialized fields and disciplines, such as feelings of fear, inferiority, jealousy, envy, humiliation, and anger. We analyze situations in which these emotions emerge and the kinds of behavior they motivate in the context of scientific imperialism.

Status and resource asymmetries among disciplines are a common source of emotional tensions in interdisciplinary collaborations. Natural and life sciences generally enjoy higher status than social sciences and humanities for reasons that seem obvious to some and contestable to others. These asymmetries influence the work of interdisciplinary research teams even if they operate under the rhetoric of mutuality, reciprocity, and exchange. Social scientists and humanists may feel subjugated and weak as they typically need to learn more about the disciplinary perspectives of life scientists than these will learn about the viewpoints of social scientists and humanists (see Callard \& Fitzgerald 2016). For example, social science and humanities researchers working in medical faculties have been forced to adjust to the research evaluation criteria of medical research that significantly differ from their own disciplinary standards, with ensuing feelings of frustration, despair, and alienation (Albert et 
al., 2014). Feelings of subjugation may also emerge from scientific subordination in which a discipline or field co-opts the explanatory and empirical practices of another discipline (or its individual researchers) to service its own supposedly more productive theories (MacLeod, this volume). Further still, natural scientists (and economists among the social scientists) enjoy a higher prestige in society as politicians and policy makers tend to seek their advice in practical problems that have technical and measurable dimensions, however richly social these problems otherwise might be. Facing these asymmetries of prestige and power, social scientists and humanists can either respond with anger, trying to achieve more recognition for their substantial efforts and contributions as Rabinow (2009) suggests, or, if they take a more pragmatic approach as Callard and Fitzgerald (2016) recommend, acquiesce in their subjugated position, experiencing occasional "sadomasochistic" pleasures in it. Both choices come with disciplinary emotions of different kinds.

A most consequential context of disciplinary emotions in interdisciplinary interaction is expansionist scientific imperialism. One way for it to begin is when one discipline seeks to explain phenomena that are perceived to belong to the domain occupied by another discipline. 'Belonging' and 'occupying' are not easy concepts to analyze, but roughly, the idea is that the identity of many disciplines is partially constituted by having certain types of phenomena in their explanatory purview on historical grounds, their having made regular attempts to explain those phenomena, or at least having them programmatically on their explanatory agenda, by including them in the scope of the discipline. Therefore, one discipline's attempt to expand the applicability of its methods, concepts, or theories to explain phenomena in another discipline's domain can be perceived as unwelcome intrusion across disciplinary boundaries, especially if the expanding discipline defines the new domain in its own disciplinary terms, suggesting that the domain 'belongs to' its 'territory'. This kind of activity can be described as a form of scientific imperialism: imperialism of scope. In its weakest and apparently most 
innocent form, imperialism of scope amounts to domain-only imperialism in which an imperialist discipline merely seeks to explain phenomena in domains that belong to other disciplines, without any attempt to change the ways of inquiry in these disciplines. In the more intrusive and far-reaching forms of imperialism, those of style or of standing, the targeted discipline itself - its principles and practices, or its status - is challenged more seriously, by transferring or imposing the research styles and strategies of the imperialistic discipline on other disciplines, and/or by appropriating the academic resources of the imperialized disciplines (Mäki 2009, 2013). One would expect the latter two forms of imperialism to trigger the strongest emotional responses, among scientific imperialists as well as among the practitioners in the imperialized disciplines.

Disciplinary emotions of the imperialists

Scientific imperialism associates with feelings of confidence, superiority, and pride in the discipline's theories, methods, and research styles, especially in relation to other disciplines, among its adherents. The feelings of confidence and superiority may have multiple sources. Thus Marion Fourcade and her co-authors (2014) argue that the perceived superiority of economics springs from sources like these: (1) its relative insularity as a discipline and its dominant position within the network of social sciences; (2) a pronounced hierarchy within the discipline, especially in comparison with other social sciences; (3) changing networks of affiliations of economics in consequence of which economics has reoriented towards finance; and (4) the material situation, worldviews, and social influence of economists that have set economists apart from their academic peers. These factors reinforce each other such that the "position of social superiority breeds self-confidence, allowing the discipline to retain its 
relative epistemic insularity over time and fueling a natural inclination towards a sense of entitlement" $(2014,2)$.

Focusing on epistemic sources of superiority that belong to the disciplinary ethos, economists believe that their discipline is the most scientific among social sciences by virtue of its mathematical methods that are more reliable and intellectually demanding than the methods of other, less formal, social sciences. With this pride in the superiority of their discipline, economists "are less likely to feel the need to rely on other disciplines or even acknowledge their existence" (Fourcade \& al. 2014, 6). Instead, economists are eager to apply their theories and methods to the study of phenomena that have traditionally belonged to the domains of other disciplines. In a famous defense of economics imperialism, Edward Lazear proudly proclaims that "by almost any market test, economics is the premier social science... [as] the economic toolbox can be used to address a large variety of problems drawn from a wide range of topics" (Lazear, 2000, 99).

It is notable that this appeal to the "market test" establishing the supremacy of economics is an exercise of (higher order) economics imperialism: it applies the notion of the market to the justification of the interdisciplinary spread of the notion of market (together with other economic notions) to traditionally non-economic domains. Rather than invoking principles such as those of evidential support or ontic similarity, it emphasizes the influence of economics on others outside of economics: "The effort to extend the field measures its success by inducing others to adopt the economic approach to explore issues that are not part of classical economics" (Lazear, 2000, 104). By this criterion, the imperialist expansion of economics to the domains of other disciplines justifies itself! Lazear grants that other scientists such as sociologists, anthropologists and perhaps psychologists may be better than economists in identifying problems of research. However, this recognition is half-hearted as economics is claimed to be superior to those other disciplines in its capacity to provide 
concrete solutions to these problems as "the parsimony of our method and ability to provide specific, well-reasoned answers gives us a major advantage in analysis" (Lazear, 2000, 103).

\section{Disciplinary emotions of the imperialized}

The picture is considerably different in the imperialized disciplines. Focusing here on externally driven intrusions (rather than internally driven importations) ${ }^{5}$, they tend to prompt emotions such as fear, jealousy, envy, humiliation, and anger. Jealousy derives from the fact that disciplines have distinct research domains that on historical grounds or otherwise appear to belong to the discipline. Even if jealousy is typically perceived and analyzed as an interpersonal emotion (e.g. Rorty, 1980; Taylor, 1988; Goldie, 2000), it may have organizational forms that are importantly similar to paradigmatic interpersonal cases. Just as interpersonal jealousy involves treating the loved one as a possession, disciplinary jealousy involves possessive inclinations about the discipline's traditional or customary domain. Accordingly, members of a vulnerable discipline may guard the discipline's domain and borders jealously against perceived or anticipated invasions from other disciplines. Jealousy motivates the building of symbolic and institutional walls around the discipline's domain in order to keep intruding scientific imperialists away. An unintended and undesirable effect of such a strategy may be the rejection of innocent, non-imperialistic proposals for interdisciplinary collaboration as well.

The main problem with disciplinary jealousy, as with interpersonal jealousy, lies in the idea of exclusive possession. Disciplinary domains cannot be owned in a literal sense any more than people. Tradition alone cannot give a sufficient justified reason for excluding researchers from other disciplines from attempting to provide alternative solutions to the 
problems of one discipline with their own methods, theories, and concepts. In an ideal situation, researchers from all sides should have the right to defend their disciplinary approaches in an interdisciplinary debate where the best argument should win. Naturally, there is no guarantee that the discipline whose traditional target phenomena are being studied with the resources of another discipline emerges as the winner from such a challenge. Therefore, disciplinary jealousy - like its interpersonal counterpart - comes with a sense of powerlessness regarding the agent's ability to keep actual or potential rivals away. This awareness generates and maintains fear and insecurity among members of vulnerable disciplines who feel threatened by the advances of imperialist disciplines into their field.

The propensity for disciplinary jealousy may be associated with the permeability of disciplinary boundaries. Becher and Trowler (2001) suggest that disciplines differ from each other in this respect.

Impermeable boundaries are in general a concomitant of tightly knit, convergent disciplinary communities and an indicator of the stability and coherence in the intellectual fields they inhabit. Permeable boundaries are associated with loosely knit, divergent academic groups and signal a more fragmented, less stable and comparatively open-ended epistemological structure (Becher and Trowler 2001, 60).

In other words, relatively insulated, hierarchical, and methodologically or theoretically unified disciplines, such as economics, are capable of protecting their borders more effectively than more open, less hierarchical, and internally diverse disciplines. We may then surmise how these characteristics are related to dispositions of disciplinary jealousy. On the one hand, the permeability of a disciplinary border may be associated with a higher propensity for disciplinary jealousy because it renders the discipline chronically susceptible to external 
influence. Yet on the other hand, and more plausibly, methodological and theoretical pluralism within a discipline may be an antidote against this emotion. Therefore, we may conjecture that the highest propensity of disciplinary jealousy is likely to be found in relatively convergent, methodologically unified disciplines such as anthropology whose domain is targeted by other equally convergent disciplines, such as economics, with their own allegedly superior methods.

If disciplinary jealousy is felt in response to perceived or anticipated intrusions into a disciplinary domain, disciplinary envy is more often felt about issues of power and standing academic and institutional resources and positions. Callard and Fitzgerald (2016), for instance, report the "neuroscience envy" of social scientists and humanists, focusing on power and resource disparities between their disciplines and the neurosciences. It is also possible to envy another discipline's methods and research styles on disciplinary grounds, that is, to regard those of one's own discipline as inferior to the envied discipline. An example is the "physics envy" of economists that according to Mirowski involves "a certain contempt for the history of economics, a tendency toward an uncritical appropriation of a limited range of mathematical formalisms, and constant intrusions by physical scientists seeking to upgrade the scientific status of the discipline" $(1992,61)$. The self-image of economists as "more scientific" and "more rigorous" than other social scientists derives from this emulation of mathematical physics that "rests almost entirely upon superficial points of resemblance between physics and economics" $(1992,67)$. Insofar as economists have successfully convinced others of their scientific status, their disciplinary envy has brought them a standing and status that members of other social science disciplines now envy in turn for the economists' position as influential and highly paid experts in business and politics.

There is another possible contemporary source of disciplinary envy that also reveals one of the internal tensions in contemporary science policy. The tendency of decreasing 
public funding of research and higher education in European universities may increase disciplinary envy as different disciplines start perceiving each other as adversaries in zerosum games. At the same time, it is recognized that addressing today's grand challenges requires interdisciplinary efforts, and research policies are designed to promote close collaborations between disciplines. The left and right hand of science policy may thus be pulling in opposing directions in this respect.

Epistemic injustice is a source of many negative disciplinary emotions that may be triggered in interdisciplinary situations. Miranda Fricker (2007) who introduced this term did not associate it with interdisciplinary interaction, but used it more generally to characterize the connection between social and epistemic power in the creation and maintenance of social meanings. She maintains that these social processes often work against those in otherwise marginalized positions in society, such as women and sexual and ethnic minorities. She distinguishes between two forms of epistemic injustice: hermeneutical and testimonial. The former is “the injustice of having some significant area of one's social experience obscured from collective understanding owing to hermeneutical marginalization" $(2007,158)$. Fricker argues that hermeneutical injustice is wrong because it results in the victim's loss of confidence in his or her beliefs and their justification or - in the extreme - loss of knowledge. Testimonial injustice has the same consequences even though it operates differently: through prejudice that causes a hearer to give a speaker less credibility as a knower than he or she deserves.

Fricker identifies feelings of humiliation and under-confidence elicited by being a victim of epistemic injustice. These feelings are intelligible in the face of imbalance of social power between the perpetrator and the victim of epistemic injustice that render the latter incapable of resisting or protesting against his or her unjust treatment. Yet if we consider an individual in a more equal power position, we should not be surprised if she perceives 
epistemic injustice as offence or insult, and responds with anger instead. Anger is felt in response to perceived offences, slights, and wrongs, as philosophers of emotion from Aristotle onwards have pointed out. Fricker does not mention anger as an emotional response to epistemic injustice, and the reason for this is that in her understanding, the socially and epistemically marginalized groups lack moral and epistemic resources to perceive their treatment as unjust in the first place. ${ }^{6}$

The situation is different in many contexts of scientific imperialism where the members of an imperialized discipline often perceive the imperialists' intrusion into their domain as an offence, sometimes prompting them angrily to resist it. If active resistance will not arise, feelings of humiliation, despair, and under-confidence are among the possible other responses. The Canadian social scientists and humanists who work in medical faculties are a case in point as testified by their dire feelings about their inability to resist the evaluation of their research practices, productivity, and academic excellence in terms of disciplinary criteria alien to them (Albert et al., 2014). These scholars are victims of testimonial injustice as they are evaluated by medical researchers whom they perceive to lack the expertise to soundly evaluate their work. Yet it seems evident that the feelings prompted by epistemic injustice include feelings of anger besides those of humiliation and despair.

Disciplinary anger can be felt in interdisciplinary interaction when members of a discipline appraise that their discipline or disciplinary colleagues have been treated unfairly or harmed in some other way by members of another discipline or by administrators, funding agencies or structural conditions unduly favoring the other discipline. The difference between anger and jealousy is that the latter is felt typically when an offensive action is merely anticipated or conceived in imagination, whereas anger presupposes an actually experienced action of another party that the subject(s) of emotion appraises as an offence of some kind. Testimonial injustice is in operation in scientific imperialism insofar as members of an 
imperialistic discipline seek to undermine the credibility of researchers of another discipline as experts in the relevant domain (Rolin, this volume). Expertise is a matter of competence in a particular domain, and credibility is others' perceived view of another subject's competence. Scientific imperialism in which the expertise available in another discipline is either ignored or regarded as categorically inferior to that of the imperialist discipline amounts to testimonial epistemic injustice.

Two further observations are in order. First, different sets of disciplinary emotions are often associated as the two sides of a skewed imperialist coin. For example, insofar as scientific imperialism is motivated by disciplinary pride, it appears that anger of the target and pride of the proponent function as complementary disciplinary emotions. Second, the disciplinary emotions of both scientific imperialists and their targets are rationally grounded in the disciplinary ethos of the respective disciplines. The disciplinary anger of those who perceive themselves as victims of epistemic injustice derives from the ethos of their discipline that they perceive as being violated by scientific imperialists who claim to have superior methods or theories for studying the relevant phenomena. Likewise, disciplinary envy and anger are grounded in the disciplinary ethos of the imperialized disciplines as these emotions relate to conceptions of research styles and proper domains of disciplines.

Besides first-order epistemic and methodological norms and strategies, the disciplinary ethos may include second-order judgments about the superiority of those norms and strategies in dealing with wider sets of phenomena or problems than those traditionally reserved for the discipline in an academic division of labor. Confidence in the superiority of the discipline's theories and research styles can be accompanied by a Kipling principle which states that it is the superior discipline's burden to bring scientific enlightenment and progress to other, less advanced disciplines (Mäki, 2013). Insofar as the imperialists have positive emotions about their first-order epistemic and methodological principles and strategies, these 
emotions easily attach to their second-order judgments about those first-order standards as well. This affective investment has the potential of reinforcing the capacity of these secondorder judgments to help resist or ignore possible evidence against these judgments, thus strengthening scientific imperialists' ${ }^{\prime}$ confidence in their mission.

Economics and evolutionary biology are examples of disciplines with this kind of self-appointed mission: economics by virtue of its mathematical methods of modelling that economists regard as superior to those of other social sciences with less formal methods, and evolutionary psychology relying on its theoretical view that human behaviors can be explained as adaptations formed at the dawn of humankind (Barkow, Cosmides \& Tooby, 1992). In contrast to these overtly imperialistic disciplines, some disciplines and theories such as gender research and social constructivism can be covertly imperialistic even if they do not recognize this pattern due to their particularistic and subversive meta-understanding of their own epistemic perspective, which may let these researchers perceive themselves as victims of other kinds of scientific imperialism. Yet those researchers' own imperialistic inclinations manifest in their confidence in expanding their analyses to ever new social and cultural phenomena and in their belief that these analyses are superior to the previous ones.

\section{The justification of disciplinary emotions}

A major philosophical question about disciplinary emotions in the context of scientific imperialism concerns their rational justification. Disciplinary ethos with its first-order epistemic and methodological norms and second-order judgments about the validity and applicability of those norms provides an internal justification for disciplinary emotions within the disciplinary community. Unfortunately, this intra-disciplinary perspective is not very 
useful in interdisciplinary contexts. Insofar as a given disciplinary ethos is internally coherent, it seems capable of justifying those same emotions that we have already discussed as examples of disciplinary emotions in imperialistic contexts: anger at epistemic injustice experienced by victims of scientific imperialism, and pride of its proponents in the superiority of their methods and research styles. Jealousy is a borderline case because it is possible to criticize the belief that a discipline has a monopoly over its domain as misguided even if such a belief is part of the disciplinary ethos. Here we point out the possible mistake regarding what can and cannot be determined by a disciplinary community on the basis of an intradisciplinary rational consensus.

Therefore, the justification of disciplinary emotions is not merely an intradisciplinary issue, but reaches beyond disciplinary boundaries. Obviously, this makes it an essentially harder problem to tackle. Nevertheless, the way disputes often go between scientific imperialists and their opponents suggests that it is impossible to avoid putting the issues more broadly as relational interdisciplinary issues. Are the imperialists' feelings of pride, superiority, and confidence manifestations of a collective hubris (Sullivan \& Hollway, 2014) and hence categorically inappropriate versions of these feelings, involving aggression and narcissistic derogation or belittling of other disciplines and their members? Or are scholars in the target disciplines overly timid and sensitive in jealously guarding their domain, or in taking offence at sincere attempts to bring epistemic progress in their field? We propose that in answering these tricky questions one needs to consider the ontological, epistemological, axiological, and institutional constraints on scientific imperialism that one of us (Mäki, 2013) has proposed elsewhere. The key idea is that in order to be acceptable, an imperialistic expansion or intrusion must meet these constraints.

The ontological constraint requires that the accomplishment of scientific imperialism should be ontological unification of apparently diverse kinds of phenomena using the 
theoretical and methodological resources of the imperialistic discipline. The successful pursuit of ontological unification is a process of discovery of real unity in the world. It is a discovery of facts pertaining to the extent to which the domains of different disciplines are made of similar components or governed by similar laws or causal mechanisms. Given that it is the task of science to make discoveries about the real world, it should be appropriate to try to unify as far as possible, regardless of whether disciplinary boundaries need to be crossed.

The epistemological constraint derives from the acknowledgement of significant epistemic uncertainty in interdisciplinary trespassing. There is no perfect assurance that ontological unification has been achieved when attempted. Epistemic caution and humility is therefore required when accepting and rejecting theories and explanations of a discipline especially when applied to far away territories. Epistemologically appropriate imperialistic claims about the cross-disciplinary unity of phenomena must not hide from view the uncertainties involved but is rather obliged to make them visible by stating the relevant provisos explicitly.

The axiological constraint gives non-epistemic values a role to play. Judgements about success and failure in unification must be made relative to values that express the human or cultural significance of the unified phenomena. Theories that manage to unify insignificant phenomena while ignoring or concealing significant ones are less justifiable than those that manage to unify significant phenomena. The concern about important human or cultural values that may fail to be expressed and may be suppressed as a result of scientific imperialism (see Clarke and Walsh 2009) is a reason for putting forth the axiological constraint.

The institutional constraint is the requirement that the pursuit of cross-disciplinary unification must occur under the guidance of the principles of institutionally virtuous scientific practice. The ideal normative institutions of honorable science should advise for 
virtues such as respectful humility and against vices like dismissive hegemonic arrogance in interdisciplinary relations. Such institutions prescribe a culture of critical and open-minded conversation sustained by suitable degrees of diversity and tolerance, and they proscribe against dogmatism and intellectual monopolies that are protected by non-argumentative means of exclusion. Scientific imperialism that engages in critical and respectful debate and that spells out and seeks to justify its presuppositional posits is more agreeable than one that marches on just by relying on the academic or non-academic standing of the invasive discipline.

We are now ready to propose that the justification of disciplinary emotions in the context of scientific imperialism should be evaluated in terms of their adaptiveness in promoting epistemically acceptable cross-disciplinary unification that meets the above four constraints. We recognize that it may be difficult to evaluate whether a particular attempt of imperialistic expansion satisfies this criterion when the intellectual space is filled with imperialist arguments and their counterarguments. Moreover, it seems obvious that the parties of such interdisciplinary disputes may not be the best judges in this question when their disciplinary emotions run high, influencing their beliefs and evaluative judgments in ways that make their respective emotions appear justified for themselves in the situation. Jealousy in particular is notorious for influencing thinking and imagination in ways that motivate the subject of emotion to act on his or her emotional thoughts. Similarly, disciplinary jealousy may make its subjects dwell on possessive thoughts about what is perceived as a hostile imperialistic invasion of their discipline, thus predisposing them against all (including reasonable) proposals of cross-disciplinary unification or even non-imperialistic collaboration. In a like manner, the pride of scientific imperialists tends to influence their thinking in ways that support their confidence that the necessary constraints of cross-disciplinary unification are being met, whether or not that is actually the case. 
Even if difficult, we believe that it is possible to give some guidelines on the basis of the four constraints. ${ }^{7}$ Imperialism of scope in its domain-only variety seems the most innocent and potentially supportable form of imperialism. No domain of phenomena is the sole property of any discipline, so attempts by neighboring disciplines to explain those phenomena should not be resisted. Accordingly, disciplinary emotions that express this kind of resistance such as anger, jealousy, and fear about such activity should be taken as conditionally warranted at most. Yet the situation is complicated by the fact that the actual justification of these emotions depends on whether imperialism of scope satisfies the ontological, epistemological, axiological, and institutional constraints. Imperialism of style and standing are even more difficult to assess. Imperialism of style may in principle inspire the target discipline to self-examine and improve its methods and standards of inquiry, but it may also lead to an underestimation or even demolition of approaches and styles of inquiry that are valuable in meeting some important epistemic or human demands. Likewise, imperialism of standing may be based on misusing weakly justified images of academic prestige so as to enhance the academic or societal status of a discipline at the expense of that of another. Or, in some (lucky but perhaps rare) cases, it may be a way of reallocating institutional resources and power positions in a way that reflects the actual epistemic capacities and performances of the disciplines involved.

Passing judgement on these matters is highly fallible and time-consuming. Therefore, it should take place in circumstances that support criticism and self-criticism, tolerance and pluralism, mutual respect and understanding. Disciplinary emotions should be so tuned that they do not prevent these considerations but rather facilitate them. The problem is that this kind of cognitively complex and future-oriented perspective of deliberation is difficult for emotions to achieve. This is because of the evolutionary function of emotions is to motivate spontaneous action in their eliciting situations: fleeing in fear, hiding in shame, attacking in 
anger, and so on. It is possible to employ the motivational force of emotions to other, more distant purposes, but this requires their reflective regulation (Gross, 1998).

Here the challenge is to regulate our disciplinary emotions on the basis of our understanding on the epistemic acceptability of particular instances of scientific imperialism. Our advice is to treat disciplinary emotions as "fire alarms" that detect situations where we have important concerns at stake but where collective reflection within the disciplinary community is required in order to determine whether or not the prima facie emotional appraisal of the situation was justified. This is not an easy task, but perhaps still feasible as the example of social and political movements suggests. The joint action in such movements is often motivated by the participants' shared emotions, and even if these emotions fade during collective deliberation on the suitable course of joint action, it is possible to reinvigorate the emotion when an opportunity to act on the emotion emerges (e.g. Salmela \& Nagatsu, 2016). Researchers on interdisciplinarity, philosophers of science in particular, have an important role to play here thanks to two advantages: they can aspire to function as neutral experts who do not have stakes in disciplinary disputes between scientists; and they are equipped with the capacity of offering diagnostic analyses of various hard cases to discern presuppositions that may obstruct fruitful interdisciplinary encounters. In principle at least, philosophers are in a position to judge whether constraints on cross-disciplinary unification and collaboration are being observed in actual projects of interdisciplinary transfer or integration. A little more elaboration is needed in the framework we have outlined here to help philosophers succeed in the difficult task. This is a challenge for another study. 


\section{Final thoughts}

In this chapter, we have presented the first theoretical account of disciplinary emotions and their role in interdisciplinary interaction, focusing on scientific imperialism. In many characteristic instances - especially those governed by externally driven intrusions - the situation emerges as asymmetrical. Members of disciplines targeted by scientific imperialism feel inferiority, jealousy, envy, humiliation, anger, and other negative emotions about their position, whereas imperialists enjoy feelings of superiority, confidence, and pride. We suggested that the justification of disciplinary emotions in the context of scientific imperialism should be evaluated in terms of their adaptiveness in promoting epistemically acceptable cross-disciplinary explanatory unification that meets the ontological, epistemological, axiological, and institutional constraints. Finally, we put forward an idea that philosophers of science may be in a relatively good position to act as neutral arbitrators capable of adjudicating in disciplinary conflicts about the acceptability of instances of scientific imperialism - and, consequently, about the justification of disciplinary emotions in those situations.

We close with two final and perhaps more sanguine observations. First, our focus on the externally driven variants of scientific imperialism has resulted in emphasizing negative emotions like fear and anger within the target disciplines. Internally driven variants - acts of importation carried out by practitioners within the target disciplines - are likely to be associated, at least in somedisciplinary circles, with positive feelings of hope and excitement, joy and curiosity, superiority and confidence. Second, interdisciplinary interaction can take mutually rewarding forms as well. There is evidence that successful non-imperialistic interdisciplinary collaboration can produce high levels of emotional energy that imbues the collaborators with confidence, enthusiasm, and strength for scientific work, while reinforcing 
their bonds of solidarity (Boix Mansilla et al., 2015; Parker \& Hackett, 2012; Collins, 1998). Importantly, these positive emotions are intertwined with cognitive and interactional dimensions of research collaboration. Thus, collective excitement and joy are markers of establishing an intellectual common ground emerging from different disciplinary viewpoints as well as of the formation of shared group identities. Some positive emotions that arise in successful interdisciplinary interaction are not disciplinary. Instead, they are interdisciplinary as they reflect an expansion of the researcher's academic identity from a disciplinary perspective to an interdisciplinary one that is experienced as rewarding and empowering (Turner et al., 2015). This finding provides a nice closing to this chapter as it highlights the substantial role of emotions in all forms of interdisciplinary interaction.

\section{Acknowledgements}

We thank Manuela Fernandez Pinto and Adrian Walsh for their insightful comments on an earlier version of this chapter.

\section{References}

Albert, Mathieu, Elise Paradis, and Ayelet Kuper. 2014. "Interdisciplinary Promises Versus

Practices in Medicine: The Decoupled Experiences of Social Sciences and Humanities

Scholars." Social Science \& Medicine 126: 17-25.

Barbalet, Jack. 2011. "Emotions Beyond Regulation: Backgrounded Emotions in Science and Trust." Emotion Review 3: 36-43.

Barkow, Jerome H., Leda Cosmides, and John Tooby. 1992. The Adapted Mind. Oxford: Oxford University Press. 
Becher, Tony and Paul R. Trowler. 2001. Academic Tribes and Territories. Buckingham: The Society for Research into Higher Education \& Open University Press. $2^{\text {nd }}$ Edition.

Ben-Ze'ev, Aaron 2000. The Subtlety of Emotions. Cambridge (MA): The MIT Press.

Bloch, Charlotte. 2012. Passion and Paranoia. Emotions and The Culture of Emotion in Academia. Aldershot: Ashgate.

Boix Mansilla, Veronica, Michelle Lamont, and Kyoko Sato. (2015) “Shared CognitiveEmotional-Interactional Platforms: Markers and Conditions for Successful Interdisciplinary Collaborations." Science, Technology \& Human Values, 1-42. DOI: 10.1177/0162243915614103

Callard, Felicity and Des Fitzgerald. 2016. Rethinking Interdisciplinarity Across the Social Sciences and Neurosciences. Basingstoke: Palgrave Macmillan.

Clarke, Steve and Adrian Walsh. 2009. "Scientific Imperialism and the Proper Relations Between the Sciences.” International Studies in the Philosophy of Science 23: 195-207. Collins, Randall. 1998. The Sociology of Philosophies. Cambridge: Harvard University Press. Davidson, Donald. 1982. "Paradoxes of Irrationality." In Richard Wollheim and Jay Hopkins. (eds). 1982. Philosophical Essays on Freud. Cambridge: Cambridge University Press, pp. 289-305.

Döring, Sabine A. 2009. “The Logic of Emotional Experience: Noninferentiality and The Problem of Conflict Without Contradiction”. Emotion Review 3: 240-247.

Elgin, Catherine Z. 2008. “Emotion and Understanding.” In Georg Brun, Ulvi Doĝuoĝlu and Dominique Kuenzle. (eds). 2008. Epistemology and Emotions. Aldershot: Ashgate, pp. 33-50. Fleck, Ludwik. 1979[1935]. Genesis and Development of a Scientific Fact. Translated by Fred Bradley and Thaddeus J. Trenn. Chicago: University of Chicago Press. 
Fourcade, Marion, Etienne Ollion and Yann Algan. 2014. “The Superiority of Economics.” maxpo discussion paper 14/3. Max Planck Sciences Po Center.

Fricker, Miranda. 2007. Epistemic Injustice: Power and the Ethics of Knowing. Oxford: Oxford University Press.

Gross, James J. 1998. “Antecedent- and Response-Focused Emotion Regulation: Divergent Consequences for Experience, Expression, and Physiology.” Journal of Personality and Social Psychology 74: 224-237.

Helm, Bennett. 2008. "Plural Agents." Nous 42: 17-49.

Henkel, Mary. 2000. Academic Identities and Policy Change in Higher Education. Higher Education Policy Series 46. London and Philadelphia: Jessica Kingsley Publishers.

Hookway, Christopher. 2008. "Epistemic Immediacy, Doubt, and Anxiety: On a Role for Affective States in Epistemic Evaluation.” In Georg Brun, Ulvi Doĝuoĝlu and Dominique Kuenzle. (eds). 2008. Epistemology and Emotions. Aldershot: Ashgate, pp. 51-66.

Koppman, Sharon, Cindy L. Cain, and Erin Leahey. 2015. “The Joy of Science: Disciplinary Diversity in Emotional Accounts." Science, Technology, and Human Values 40: 30-70.

Kuhn. Thomas. 1970. The Structure of Scientific Revolutions. Chicago: The University of Chicago Press.

Lazear, Edward. 2000. “Economic Imperialism.” The Quarterly Journal of Economics 115: $99-146$.

Lyons, William. 1980. The Emotions. Cambridge: Cambridge University Press.

Mäki, Uskali. 2009. “Economics Imperialism: Concepts and Constraints.” Philosophy of the Social Sciences 39: 351-380. 
Mäki, Uskali. 2013. “Scientific Imperialism: Difficulties in Definition, Identification, and Assessment." International Studies in the Philosophy of Science 27: 325-339.

Mäki, Uskali and Caterina Marchionni. 2011. “Is Geographic Economics Imperializing Economic Geography?” Journal of Economic Geography 11: 645-665.

Mirowski, Paul. 1992. “Do Economists Suffer From Physics Envy?” Finnish Economic Papers 5: 61-68.

Parker, John N. and Edward J. Hackett. 2012. "Hot Spots and Hot Moments in Scientific Collaborations and Social Movements.”American Sociological Review 77: 21-44.

Parry, Sharon. 2007. Disciplines and Doctorates. Higher Education Dynamics, 16. Dordrecht: Springer.

Rabinow, Paul. 2009. "Prosperity, Amelioration, Flourishing: From a Logic of Practical Judgment to Reconstruction." Law and Literature 21: 301-320.

Radder, Hans. 2010. The Commodification of Academic Research: Science and the Modern University. Pittsburgh: University of Pittsburgh Press.

Rorty, Amelie. 1980. “Jealousy, Attention, and Loss.” In Amelie Rorty (ed.). Explaining Emotions. Berkeley: University of California Press, pp. 465-488.

Salmela, Mikko. 2011. “Can Emotion Be Modelled on Perception?” Dialectica 65: 1-29

Salmela, Mikko. 2012. “Shared Emotions.” Philosophical Explorations 15: 33-46.

Salmela, Mikko \& Michiru Nagatsu. 2016. "Collective Emotions and Joint Action: Beyond Received and Minimalist Approaches.” Journal of Social Ontology 2: 33-57.

Schoenberger, Erica, 2001. "Interdisciplinarity and Social Power." Progress in Human Geography 25: 365-382 
Sullivan, Gavin B. and James Hollway. 2014. "Collective Pride and Collective Hubris in

Organizations.” In Gavin B. Sullivan (ed.). Understanding Collective Pride and Group

Identity. New Directions in Emotion Theory, Research and Practice. London and New York:

Routledge, pp. 80-92.

Tanesini, Alessandra. 2008. "Virtues, Emotions and Fallibilism.” In Georg Brun, Ulvi

Doĝuoĝlu and Dominique Kuenzle. (eds). 2008. Epistemology and Emotions. Aldershot:

Ashgate, pp. 67-82.

Taylor, Gabrielle. 1988. "Envy and Jealousy: Emotions and Vices.” Midwest Studies in

Philosophy 13: 11-27.

Tuomela, Raimo. 2007. Philosophy of Sociality. New York: Oxford University Press.

Turner, V. Kelly, Karina Benassaiah, Scott Warren and David Iwaniec. 2015. "Essential

Tensions in Interdisciplinary Scholarship: Navigating Challenges in Affect, Epistemologies,

and Structure in Environment-Society Research Centers.” Higher Education 70: 649-665

\footnotetext{
${ }^{1}$ Yet see Parker \& Hackett's (2012) discussion on Fleck's ideas.

${ }^{2}$ Donald Davidson (1982) famously argued that a person's individual beliefs, desires, intentions, and actions as well as many emotions are intelligible only insofar as they fit into a coherent and holistic pattern that also determines the rationality of the individual constituents of the pattern. The idea is that we for instance fear what we believe to be harmful to something we value, and rationality amounts to this kind of coherence among an agent's attitudes and responses. It is possible to apply this view to social groups as well. The idea is that a social group may manifest a shared evaluative perspective in the group members' responsiveness to the world, and these responses are rational by virtue of their fitting into the coherent pattern (Helm 2008, Salmela 2012). For instance, if a group has set for itself the task of painting a house, then the group members should exhibit a coherent pattern of hope, fear, frustration, anticipation, disappointment, joy, relief, and so on in the appropriate conditions and for the right reason that goes back to their goal of painting the house. Some philosophers have argued that the rationality of emotions cannot be evaluated in terms of their coherence with the agent's beliefs and evaluative judgments because emotions are not inferentially related to those attitudes but instead logically similar to sense perceptions (e.g. Döring 2009). However, this argument fails because it is possible to bring normal human emotions in line with beliefs and evaluative judgments, sometimes with time and efforts, whereas this is impossible for sense perceptions as the recalcitrant Müller-Lyer visual illusion shows (Salmela 2011). ${ }^{3}$ There is a lively theoretical debate among philosophers on what it means for an emotion to be shared or collective. The two main theoretical approaches are type-identity and token-identity views. According to typeidentity views, two or more individuals share an emotion if each of them has an emotion of the same type such as anger or fear about the same object or event. According to token-identity views, there is a single emotion with several participants, similarly to there is a single car with several passengers who share a ride. In this chapter, we adopt a type-identity view of emotional sharing. For an argument for this view, see e.g. Salmela (2012).
} 
${ }^{4}$ Bloch (2012) points out that other motives besides disciplinary ones may become mixed with a reviewer's anger in peer review: "Assessor anger can be a morally legitimate emotion, but it can also be an anger that mobilizes for opposition, a personally motivated feeling of anger, and it can be a group emotion that prepares the ground for negative or less respectful assessments under the guise of an academic sense of justice" (Bloch, 2012, 60). We do not disagree but we focus here on cases of the first kind in which anger - and other emotions in peer review - are justified by disciplinary criteria of scholarly excellence alone.

${ }^{5}$ This distinction is between intrusion into a target discipline or its" domain from outside carried out by an expansionist discipline; and importation of items from an expansionist discipline carried out by practitioners active within the target discipline (Mäki 2013). The emotional dynamics is likely to be different in the two types of case. We simplify matters by focusing on the externally driven case.

${ }^{6}$ The history of different civil rights movements - black, women and gay, for instance - shows that it has been both necessary and possible for these both socially and epistemically marginalized social groups to develop an understanding of their treatment as unjust, while still being treated that way by the majority. Moreover, a central aspect of their success has been the transformation of low-energy, reactive emotions like humiliation and shame that are reflections of the oppression into high-energy, active emotions such as pride and anger that reflect an affirmation of the emancipated self.

${ }^{7}$ For a more detailed discussion on these criteria and their application to the question of whether geographical economics is imperializing economic geography, see Mäki \& Marchionni (2011). 